\author{
CANCER: STRANGE ATTRACTORS AND COMPLEXITY \\ Abicumaran Uthamacumaran \\ Concordia University \\ 7141 Sherbrooke St W, Montreal, Quebec H4B 1R6 \\ a_utham@live.concordia.ca
}

\begin{abstract}
The brief perspective suggests an unorthodox interpretation of cancer dynamics in terms of strange attractors and fluid turbulence. The relevance of strange attractors in tumor growth-invasion dynamics and chemical pattern formation are suggested. Chemical turbulence, fractals and chaos are discussed as the principal characteristics of tumor ecosystems and their complex cybernetics. To conclude, cancers are presented as complex systems which may be exhibiting chaotic gene expression dynamics.
\end{abstract}

Keywords: Cancer; Complexity; Chaos; Nonlinear Dynamics; Fractals; Turbulence

\title{
INTRODUCTION.
}

Cancer remains the leading cause of disease-related pediatric death in developed countries. Perhaps a reframing of the fundamental physics driving its pattern formation and network dynamics, a paradigm shift, is required to better understand this intractable problem. The emerging field of complexity science is such a paradigm shift, a canvas for painting cancers as complex adaptive systems. A complex system is traditionally described as a system in which the emergent properties of the whole do not reduce to the sum of its interacting parts (Mitchell, 2009; Thurner et al., 2018; Ladyman and Wiesner, 2020). This Aristotelian description is a characteristic signature of the patterns and behaviors emerging in many nonlinear dynamical systems.

Nonlinearity implies a system in which the output of the system does not change in direct proportion to the change in its inputs. This may be the root cause of why descriptions of cancer processes within the fabric of nonlinear dynamics are considered unorthodox, as it is abstract and counter-intuitive to most of our sense perception. It may be relatively safe to say the decision-making of our ancestors have evolved to infer/predict cause-effect relations within natural systems on the grounds of classical (Newtonian) physics. The sense-making of our ancestors relied primarily on the reductionist paradigm to ensure their survival and reproduction - i.e., projectile motions of spears and arrows to hunt preys, periodic behaviors in climate (light/dark), elliptical orbits in celestial mechanics accounted for the seasonal cycles, etc. and these regular patterns and limit cycles were essential to maintaining their internal feedback loop systems (cybernetics) (i.e., biorhythms, circadian activity, hormonal fluctuations, neural oscillations, etc.). That is, their pattern recognition relied heavily on the anticipation of order, or more appropriately 'regularity', and hence, predictability. Nonlinear dynamics is the devil to this primitive notion, it is the contrary of everything our daily routines depend on such as order, periodicity, stationary/equilibrium, and predictability (Note: I hesitate to use the term order, as chaos can be defined as a form of causal order). This may explain why we are transitioning towards the use of Artificial Intelligence in assessing complex causal structures and patterns in data-driven systems science - machines can be trained to spot patterns that may seem strange and non-trivial to human sense perception. Complex systems science advocates the use of machine intelligence and algorithms to study emergent behaviors and patterns in nonlinear feedback systems through the looking glass of computation and information- i.e., study patterns which cannot be inferred by reducing a system to its constituents. 
Central to the study of complex emergent behaviors are chaotic systems, i.e., systems exhibiting sensitive dependence to initial conditions and perturbations (Strogatz, 2015). Chaotic systems are often intractable, i.e., in practical cases, the differential equations (if any) describing them do not have analytical solutions (i.e., non-integrable) (Strogatz, 2015). There is a particular chaotic system revered as the Holy Grail of mathematicians for centuries: the three-dimensional, Navier-Stokes equations. Fluid turbulence is the iconic representation of complex systems. Turbulence occurs in all scales from the very large to the very small; within the astrophysical, geological, ecological, biochemical, and even quantum regimes. It is observed in the heat flow of coffee cups, the motion of galactic clusters, flickering solar flares, gusts of wind, the swirls of Van Gogh's The Starry Night, predator-prey dynamics, systemic blood flows, airflow in the respiratory tract, and the paintings of children (Lorenz, 1963; Wensink et al., 2012; Arts and Culture, 2019). Fluid turbulence is universal yet finding solutions to its equations of motion remains one of the greatest unsolved problems in mathematical physics.

Herein, it is argued that the gene expression dynamics and protein-mediated patterning of cancer cells may be chaotic instead of random. Further, it is suggested the chaotic dynamics may account for their complex adaptive behaviors such as cancer stemness and protein-mediated chemical turbulence at the onset of cancer stem cell division/differentiation. The 'may' in italic implies we still severely lack experimental confirmation of these presumptions, while they are computationally and mathematically well-documented. It would be a madman's adventures in Wonderland to not only interconnect the two problems of cancer (pattern formation) and (chemical) turbulence, but to further classify them under the fundamental roadblock of computer science: the P vs. NP problem. Reconstructing the geneinteraction networks of cancer ecosystems and reverse- engineering their attractor landscapes are NPcomplete problems (Akutsu et al., 1998; Milano and Roli, 2000; Barillot et al., 2013; Songjian et al., 2016; Hajkarim et al., 2019). This will be precisely the motivation to this short perspective, to stimulate a paradigm shift in cancer research towards that of complex systems - the general characteristics and patterns of behaviors of which includes nonlinear dynamics, self-organization, criticality, emergence, fractals, and chaos, concepts inherent to the imagination and meaning-making in children. Herein, I propose the causal structures formed by fluid turbulence and cancer may have a common mathematical language: strange attractors. This communication is to be treated as a glossary of concepts and ideas pertaining to dynamical systems theory and cybernetics applicable in the study of cancer cell fate dynamics, made available to all (wholeness) systems thinkers.

\section{STRANGE ATTRACTORS.}

In dynamical systems theory, an attractor is a set of values in state-space/phase-space toward which a complex system tends to evolve for a wide variety of initial conditions (Strogatz, 2015). A strange attractor, also known as a chaotic attractor, is a type of attractor bounded with a fractal dimension in embedding phase space. Strange attractors exhibit sensitive dependence on initial conditions (chaos) and in consequence apparent randomness. That is, the trajectories within a strange attractor appear to be unpredictable and stochastic but have some pre-defined complex structure, a causal pattern.

The study of strange attractors was inspired by the search for solutions to the Navier-Stokes equations. Lorenz (1963), often referred to as the father of chaos theory, derived the Lorenz attractor - the iconic toy model for strange attractors, by studying a simplified Benard-Rayleigh convection model in terms of three-coupled first-order ordinary differential equations in attempt to model weather turbulence, a complex system. The term strange attractor was first coined by Ruelle and Takens (1971) in their investigation of the complex multi-fractal patterns observed in fluid turbulence. Strange attractors are ubiquitously observed in tumor pattern formation and cancer growth models, although they may not be referred to as such structures (probably due to differences in the disciplines of science to which one 
adheres; dynamical systems theory and oncology have become reduced to separate branches in the Newtonian paradigm). In fact, the prediction of intracellular chemical patterns within cancer cells can be thought of as forecasting weather turbulence (Yankeelov et al., 2015). That being said, the following are some examples to illustrate the plausible role of strange attractors in tumor complexity.

The Sel'kov model for glycolysis is an important metabolic pathway in which glucose is broken down to make pyruvate. The model exhibits a Hopf pitchfork bifurcation in glycolytic oscillations which may further evolve to chaotic dynamics and strange attractors (Rensing and Jaeger, 1985). As such, it may be relevant to investigate the emergence of chaos in glycolytic oscillations in cancer cells. Further, mathematical models demonstrate chaotic oscillations of a single transcription factor can be used to up-regulate certain proteins, or specific protein complexes in gene expression regulation (Heltberg et al., 2019). Chaotic attractors can also emerge in the reaction-diffusion equations of tumour growthinvasion depending on small changes in the control parameters (Itik and Banks, 2010). For instance, the Rössler attractor is an example of a strange attractor proposed to be observed in simple chemical reaction-diffusion systems (Rössler, 1976). However, the experimental detection of intracellular chaos remains vastly ambiguous (in part, due to the apparent indistinguishability from randomness).

Such bio-mathematical models of strange attractors have also been primitively reported in experiments. For example, electric cell impedance recordings were performed in rat's prostate cancers. The time-series Fourier analysis of cancer micro-motions were assessed by Takens' theorem (time-delay embedding) to detect patterns distinguishable from a random signal. The attractor reconstruction showed positive Lyapunov exponents in the phase portraits (i.e., signatures of chaos) (Posadas et al., 1996). However, the experimental investigations into intracellular chaos somewhat inhibited by the end of 1990s, possibly by dogmatic suppression (it was strange to be talking about strange attractors within cells back then). However, mathematical models of chaotic gene expression dynamics and pattern formation in cancer ecosystems still prevailed.

Ivancevic et al. (2008) showed a Lorenz-like, chaotic attractor best describes the reaction-diffusion patterns of cancer cells, as given by $\partial \varphi / \partial t=\nabla \cdot(D(\varphi, v) \nabla \varphi(v, t))$, where $\varphi$ is the density of diffusing material (cancer cells), $v$ is the velocity, $t$ is time and $D$ is the diffusion coefficient. The emergence of Lorenz-like attractors indicate cancer cell fates may be strange-attractors of the Waddington attractor landscape. As demonstrated by the mathematical model, time-delay Hopf-bifurcations on these systems can result in period-doubling bifurcations, i.e., chaotic behaviors. This can be indicative of complex adaptive behaviors such as tumor relapse (recurrence) and the emergence of aggressive cancer phenotypes (Khajanchi et al., 2018; Zheng et al., 2018; Heltberg et al., 2019).

Cancer modelling was performed using a three-body predator-prey system consisting of host, immune and tumor cells. Assuming initially logistic growth for the population dynamics, the Lotka-Volterra dynamics of the three-body problem became chaotic as denoted by the period doubling cascades in the bifurcation diagrams (Letellier et al., 2013). The bifurcation analysis revealed Rössler-like attractors with a fractal Lyapunov dimension. Letellier et al. (2013) further proposed the understanding of tumor growth and metastasis as strange attractors will pave dynamical therapies (i.e., therapies which consider the adaptive changes in cancer processes/evolution). Regardless, these mathematical findings demonstrate how attractor reconstruction algorithms, such as time-delay embedding, may be capable of tracking chaotic behaviors in chemical oscillations, such as those pertaining to the protein flows critical for cancer pattern formation. This is the key message I would like the readers to take from this synthesis - current approaches to science fundamentally lack the application of algorithms in the study of causal inference in cancer gene expression and pattern formation. 
Speaking of predator-prey dynamics as a model-system for cancer's ecological behaviors, fluid turbulence in many ways is analogous to the predator-prey dynamics of cellular ecosystems. For instance, the simulation of an ecological system was performed with three trophic levels: Nutrient, Prey and Predator, corresponding in the fluid system to laminar flow, turbulence, and zonal flow, respectively (Shih et al., 2016). The ecological predator-prey dynamics were shown to be well characterized by models of intermittent puffs within turbulent fluid flows in a pipe. The predator-prey dynamics were driven by the competition between different wavenumber (frequency) selection mechanisms suggestive of the power law decay seen in Kolmogorov's turbulence model (Shih et al., 2016). These findings collectively suggest a deeper understanding of fluid turbulence may be needed to grasp the complexity of the predator-prey dynamics and pattern formation in tumor. To approach this suggested interpretation, let us transiently explore the governing equation of motion in fluid turbulence: the Navier-Stokes Equation (NSE).

\section{THE NAVIER-STOKES EQUATIONS.}

The Navier-Stokes equation is a nonlinear, partial differential equation. For an isotropic, incompressible Newtonian fluid (i.e., $\nabla \cdot u=0$ ), it is given by:

$$
\rho\left(\frac{\partial u}{\partial t}\right)=v \nabla^{2} u-u \cdot \nabla u-\nabla P+f
$$

Where $\mathrm{u}$ is the velocity vector, $v$ the kinematic viscosity, $\mathrm{P}$ is the pressure, $\rho$ the fluid density and $f$ is the external forces (Note: Most complex fluids in biosystems are anisotropic, and heterogeneous).

The Navier-Stokes existence, smoothness and regularity problem poses: Given an initial velocity vector, elucidate the existence of a velocity field and scalar pressure field which are both smooth and globally defined that solve the three-dimensional NSE equations? (Leray, 1934). Whether the Navier-Stokes equations allow solutions that develop singularities in finite time remains an unresolved, complexity problem (i.e., P vs. NP). However, there is an immense body of experimental and theoretical works confirming the topologies of turbulent fluid flows consist of strange attractors and multifractal structures (Ruelle, 1980; 1995; Lanford, 1982; Miles, 1984; Brandstater and Swinney, 1987).

According to L.F. Richardson, turbulent motion is a superposition of eddies undergoing an energy cascade. That is, the turbulent eddies and vortices comprising of fluid turbulence bifurcate into a multifractal hierarchy of smaller eddies. However, experimentally, even the turbulence of smallest eddies is patchy and intermittent. Turbulent phenomena in fluids are characterized by strong fluctuations and power law spectra suggestive of correlations observed in critical phase transitions. In real models, unlike the predictions of Kolmogorov's isotropic turbulence, turbulence is inhomogeneous. In experiments, turbulence arises in sudden large bursts, one after the other, followed by a period of relative quiescence (i.e., intermittency). With intermittency, a multifractal scaling is observed in the frequency spectrum. Regardless, the Kolmogorov energy distribution, $E(k) d k \sim \varepsilon^{2 / 3} k^{-5 / 3} d k$ holds well in the inertial range fluid turbulence with low dimensionality, where $\varepsilon$ is the energy flux and $E$ is the energy density (Kolmogorov, 1941). The vortex lines of turbulent flows form knotted structures called vortex tangles, consisting of interwoven loops and strings, embodying the interconnectedness and irreducibility of fluid turbulence (Arnold and Khesin, 1991; Rico, 2000). The study of these complex topologies- the vortex tangles and emergent structures, are hot topics in complex systems research.

Furthermore, a continuous frequency spectrum is a signature of experimental fluid turbulence, a method which has been exploited in the chaotic attractor reconstruction of cancer models discussed in the previous section. There is no direct test to 'sensitive dependence on initial conditions' in hydrodynamical turbulence. Hence, the frequency spectra of the fluid motion can be used to detect 
strange attractors (Ruelle, 1973; 1995). Other methods include chaos detection tools such as fractal dimension analysis and (positive) Lyapunov exponents characterization on reconstructed attractors.

\section{TURBULENCE}

Turbulence furnishes a scaling problem, as to what Reynolds number produces turbulent flows varies from one system to another. By convention, the cell cytoplasm is assumed to be dominated by viscous forces due to macromolecular crowding (i.e., cytoskeletal filaments, organelles, etc.), and low Reynolds number, thereby limiting the movement of intracellular proteins and molecules to Brownian motion. However, phase-transition to chemical turbulences can occur in reaction-diffusion systems. In theory, a single- mode lateral instability can lead to chemical turbulence at the onset of pattern formation within cells (Brauns et al., 2018). As suggested by the works of Prigogine on dissipative structures, following turbulence transition, coherent patterns spontaneously emerge through diffusively coupled local equilibria (i.e., self-organization) (Prigogine and Stengers, 1984). As Prigogine states, chaos is merely a form of order in which the temporal sequence is complex. In theory, such a transition to spatiotemporal chaos can occur in intracellular protein patterning systems as well.

A simple, two-species activator-inhibitor ( $A$ and $B$ ) model of morphogenesis (cellular pattern formation) with their respective reaction coupling terms ( $f$ and $g$ ) and diffusion coefficients $D$, is given by:

$$
\begin{aligned}
& \frac{\partial A}{\partial t}=f(A, B)+D_{A} \nabla^{2} A \\
& \frac{\partial B}{\partial t}=g(A, B)-D_{B} \nabla^{2} B
\end{aligned}
$$

assuming the intracellular protein oscillations have membrane and cytosolic bound state-transitions (Turing, 1952). Such reaction-diffusion equations are used to model intracellular protein traffics, the ECM (extracellular matrix) degradative enzyme dynamics, flows of cytoskeletal proteins and focal adhesion remodelling in cancer cells (Gatenby and Gawlinki, 1996; Ramis-Conde et al., 2008; Domsuhke et al., 2014). Both in vitro and in vivo cancer models well-fit these equations where given the appropriate chemoattractant gradients, the proteins and nutrient concentrations, the ECM-stroma dynamics can be modelled as the reaction-diffusion (invasion) system characterized by: $D=$ $\nabla(-V(x, t))+r \nabla A(x, t)$. The first term is the direction (gradient) of the potential $\mathrm{V}$, the second term is the chemotactic gradient of species $A$, and $D$ is the diffusion coefficient of species $A$ (Note: same equation applies to chemical species B) (Gatenby and Gawlinki, 1996; Ramis-Conde et al., 2008).

In a continuous description of tumor cell density, viewed as an incompressible fluid, the change in number of cells $\mathrm{N}$ is given by the partial differential equation: $\frac{\partial N}{\partial t}=D_{N} \nabla^{2} N-\nabla(N \nabla E)$, where $\mathrm{E}$ is the ECM density. Considering additional parameters such as enzyme kinetic rates, oxygen supply (i.e., hypoxia conditions), glucose level, nutrient levels, etc., a specific set of differential equations characterizing the tumor ecosystem's growth and morphogenesis may be derived.

Simplified Navier-Stokes equations such as Darcy's law can be used to better model the reactiondiffusion processes underlying GBM invasion and growth (Yan et al., 2017). However, these simple models too are often intractable even when not accounting for the emergence of spatiotemporal. Critical dynamics such as tumor phenotypic plasticity, emergence of mixed/hybrid cell fates, and cell fate interconvertibility are further ignored in these models. 
Further, systemic circulation exhibits turbulence, wherein the turbulent kinetic energy of circulatory fluids increase with ageing and pathophysiology (Hojin et al., 2018). The formation of cancers in organs and their metastases demonstrates a scaling over many orders of magnitude, from exosome flow to circulating tumor cells (CTC) in blood vessel/lymphatic networks. Such complex systems comprise of multi-scale patterns of fluid flow. For instance, at the organs level, the Reynolds number may be around 1000 , in the aorta up to 10,000 , etc. How systemic turbulence influence cancer metastases remains primitively understood. In a recent study, hematopoietic iPSC (induced pluripotent) stem cells were shown to increase their production rates in systemic flows by many orders of magnitude under turbulence (Ito et al., 2018). Low levels of turbulence generated in turbines increased the levels of platelets produced by the iPSC-derived megakaryocytes significantly. Turbulence stimulated macrophage migration inhibitory factor (MIF), insulin growth factor binding protein 2 (IGFBP2) and nardilysin, accounting for the increased platelet generation. In speculation, such mechanisms may be utilized by cancer (stem) cells or their shedding exosomes/extracellular vesicles to undergo rapid clonal expansion during metastatic flow. Such findings suggest cancer stem cells of different tissues and molecular groups must be placed in turbulent flows as within this experiment, to observe what gene expression pathways are activated under systemic turbulence. Moreover, the use of turbulence to increase platelet and HiPSC stem cells may help progress with the clinical therapies in hemato-oncology.

Cancer metastasis, growth and therapy response are governed by fluid dynamics (Koumoutsakos et al., 2013; Goetz, 2018). Using shear-stress tensor in terms of viscosity and the fluid velocity gradients, focal adhesion dynamics can be investigated in cancer metastasis (Thamilselvan and Basson, 2004; Chivukula et al., 2014). The hemodynamic shear forces modulate the formation and location of pre-metastatic niches through regulating CTC flow. Microfluidic experiments on 3D ovarian cancer models show that fluidic streams induce a motile and aggressive tumor phenotype. Hence, higher fluid flow was shown to induce EMT transitions and promote aggressive phenotypes that can metastasize (Rizvi et al., 2013; Ketene et al., 2012). Furthermore, Huang et al. (2018) showed that non-laminar (turbulent) shear stress may increase the adhesive ability of cancer cells in metastatic invasion. Language in science is ambiguous and subjected to slippage - sometimes the lack of evidence is a sign of evidence, for e.g., the use of words such as aperiodic and non-laminar may imply turbulence.

The Navier-Stokes Equations (NSE) can be used to model circulatory tumor cell (CTC) dynamics. The fluid shear stresses experienced by CTC cells consist of turbulence. Blood flow velocities in the circulation system can range from 0.03 to $40 \mathrm{~cm} / \mathrm{s}$ depending on the vessel size. How individual CTCs and CTC emboli can withstand these intercellular interactions and persist in the fluid flow is not yet fully understood. However, the fluid velocities must be solved from the NSE to measure CTC dynamics (Rejniak et al., 2016). With appropriate boundary conditions, the steady, incompressible Navier-Stokes and continuity equations are solved using approximation techniques such as the finite volume method to model circulating melanoma cells and their adhesion dynamics (Behr et al., 2015). Furthermore, even Doppler imaging techniques can track turbulent blood flow in the hepatic vein portal of liver cancer patients, where the most malignant tumors had greater turbulence (Yasuhara et al., 1997). Such findings indicate an understanding of systemic fluid turbulence may be essential to paving dynamical therapies in precision oncology.

Furthermore, blood flow is often modelled using the Hagen-Poiseuille equation assuming vessels to be cylindrical pipes and incoming flows to be laminar. However, tumor vasculature does not conform to these regular structures with uniform flow patterns. Rather, the complexity of tumor vasculature and 
tissue organization can be quantified in terms of fractal dimensions, i.e., irregular surfaces exhibiting self-similarity across scales (Jain and Baish, 1998; Nasu et al., 1999). Muti-fractal analysis is a tool to study the geometry of turbulence (Sreenivasan and Meneveau, 1986; 1991). The application of fractal dimension algorithms, multifractal analysis, and percolation clustering on tumor vasculature can be used to study how treatments alter its structural complexity (Coffey, 1998; Yakhota and Sreenivasan, 2004).

Further, turbulence may play a significant role in the protein-folding of cancer-associated protein complexes. Levinthal's paradox states protein folding is seemingly an NP-hard problem with a puzzling timescale and combinatorial space complexity. Protein folding displays a problem of combinatorial spatial and temporal complexity even the best of our current computational approaches (including Deep Learning algorithms like Google's AlphaFold) would find perplexing. However, nature resolves the folding problem within seconds. A partial explanation is held within the many interactions of the folding structure. Misfolded protein aggregates have long-range interactions with many other protein complexes and are aided by homeostatic machineries such as chaperones and proteosomes. Recent evidence show protein folding may best be described by the turbulence of Kolmogorov-Richardson's energy cascade, wherein turbulent eddies, and vortices breakdown into smaller fractal hierarchies. The protein folding flows of an SH3 domain protein model exhibited a fractal nature and were filled with 3D eddies containing strange attractors, at which the tracer flow paths behaved as saddle trajectories (Kalgin and Chekmarev, 2011; Andryushchenko and Chekmarev, 2017). The SH3 domains are crucial in focal adhesion complexes regulating cancer metastasis and EMT transitions. Likewise, folding dynamics of villin subdomain HP-35 protein in a FRET experiment was shown to obey the $\beta$-model of turbulence with many orders of turbulent flow transitions for eddies in the 3D conformational space (Andryushchenko and Chekmarev, 2016; Chekmarev, 2018).

Turbulence has been observed in the flocking and swarming of soft matter systems such as collective cell migrations and active fluids. Active fluids are a rapidly evolving research field inspired by the soft matter physics of dense suspensions of motile cells and cytoskeletal proteins such as actin and microtubules (Sumino et al., 2012; Doostmohammadi et al., 2018). Their hydrodynamic interactions give rise to the emergence of meso-scale vortex patterns reminiscent of two-dimensional turbulence. Recall, abnormal cell division (cancer) is orchestrated by these cytoskeletal filaments, cell polarity complexes, etc. Cell tissues and reconstituted cytoskeletal solutions can exhibit active turbulence, where emergent scaling behaviors can be observed. Bundles of microtubules and their associated kinesin motors deposited on an oil-water interface behaved like 3D vortex tangles in a turbulent fluid (Sumino et al., 2012). 2D distortions of nematics consisting of microtubules showed quadratic variants of the NavierStokes equations in growth (Martinez-Pratt et al. 2019). Hence, our recent findings in active matter systems and complex fluids/soft matter physics demonstrate self propelled particles like proteins involved in cytoskeletal flocking, follow continuum mechanics much alike fluids, and can exhibit turbulence even at relatively low Reynolds (Marchetti et al., 2013; Baggaley, 2016).

Usually, low intracellular concentration of proteins and their corresponding dynamics are modelled as stochastic fluctuations. However, a recent theory by Halatek and Frey (2018) challenged the dogma. Computational simulations using the finite elements method (FEM) predicted chemical turbulence (spatiotemporal chaos) at the onset of protein-mediated pattern formation. Cytosolic diffusion constants $D c$ were in the order of $60 \mu \mathrm{m}^{2} \mathrm{~s}^{-1}$ in a general 1D setting, where the MinD-ATP/ADP in bulk were given by the Turing reaction-diffusion equations: 


$$
\begin{aligned}
& \partial_{t} u_{D}(z, t)=D_{c} \nabla_{z}^{2} u_{D}-F_{u_{D}} \\
& \partial_{t} u_{T}(z, t)=D_{c} \nabla_{z}^{2} u_{T}+F_{u_{T}}
\end{aligned}
$$

Where, $u D$ and $u T$ define the cytosolic density of MinD- ATP/ADP conformations and assumed the MinD-ATP binds to the membrane via nonlinear coupling rate constants. The resultant kymograms demonstrated turbulent flows at low MinE/MinD ratios. The theoretical predictions of Halatek and Frey (2018) have been confirmed experimentally (Denk et al., 2018; Glock et al., 2019). These findings must be experimentally repeated in the PAR cell polarity complexes (the mammalian equivalence of the Min proteins) and other associated cytoskeletal remodelling proteins in cancer (stem) cells during cell fate bifurcations and cell division.

According to these findings, chemical turbulence in pattern formation is used as a synonym for spatiotemporal chaos, i.e., the emergence of a broad distribution in the power spectrum and a low spatial correlation length reminiscent of the Kolmogorov spectrum. But none of these terms are strictly/unambiguously defined in the literature. The term was adapted from the work by Nobel laureate Gerhard Ertl on reactions of heterogeneous catalysis (Kim et al., 2001). According to this work, during chemical turbulence, both the amplitude and the phase of local concentration oscillations are strongly fluctuating creating spiral waves as seen in the Denk et al. (2018) data with Min proteins pattern formation. This diffusion-induced chemical turbulence is typical for oscillatory surface chemical reactions and has been observed under special conditions in the Belousov-Zhabotinsky reaction (Kim et al., 2001). Hence, in principle, turbulent chemical oscillations can occur in cellular patterning (reactiondiffusion) systems - a concept most of our current approaches to studying cancer dynamics fails to account for (Mecke, 1996). Note: the Belousov-Zhabotinsky reaction is often a toy-model system for turbulent pattern formations (Ouyang and Swinney, 1991; Ruelle, 1995).

On a final note, keep in mind the Reynolds number is a dimensional analysis argument making the physics of fluids messy and incomplete, with only partial bits of information to connect the puzzle. In a cylindrical tube, whether we consider the length scale as the radius or diameter will change the Reynolds number by a factor of 2 . The critical Reynolds number were shown to be in the order of 100200 for Taylor-Couette and Rayleigh- Benard convection systems, which is relatively feasible in/around cellular biosystems (Ruelle, 2012; 2014).

\section{CONCLUSION}

As mentioned, there are no efficient algorithms for the detection of strange attractors in cancer ecosystems, whether it be the time lapse imaging of intracellular protein patterns or predicting cell fate dynamics on the Waddington attractor landscape reconstructed from time-series gene expression datasets. The detection of strange attractors even in fluid turbulence is restricted to experimental mapping techniques such as power/frequency spectra analysis, attractor reconstruction, fractal dimension analysis, entropy measures, and phase portraits (Lyapunov spectra). Takens mentioned entropy measures and multi-fractality as general techniques for strange attractor detection in turbulent systems (Takens, 1980). However, attractor reconstruction methods such as Takens' time-delay coordinate embedding used to achieve these results may not be well-suited for large complex datasets with many interacting variables (e.g., gene expression datasets). Further, distinguishing noise from chaos remains a fundamental roadblock in cancer biology. As such, the following are thus a few algorithmic prospects to overcome this challenge. 
Artificial Intelligence (AI) is emerging as the most powerful tool available in deciphering cancer networks, without the many biases, we humans have. Even simple machine learning algorithms such as decision trees can help distinguish noise from chaos in biological signals (Toker et al., 2020). As such, two additional $\mathrm{Al}$ algorithms are proposed herein as heuristics (approximation tools) to map strange attractors in complex, chaotic datasets: artificial neural networks (e.g., recurrent neural networks, ensemble Deep Learning Networks (DLN), etc.), and complexity measures from algorithmic information dynamics (AID).

Deep Learning architectures are the current state-of-the-art approaches in pattern recognition from complex datasets. Hopfield networks and Deep learning architectures can predict irregular patterns observed in complex fluid flows as well (Kutz et al., 2017; Yang and Huang, 2016). Strange attractors have been mapped in recurrent neural networks and gene networks (Mestl et al., 1996). Ling et al. $(2015,2016)$ first-demonstrated the applicability of Deep Learning Networks (DNN) to predict the turbulent flows of the Reynolds-averaged Navier-Stokes equations. A Galilean-invariance embedded, DNN network architecture (Tensor Basis Neural Network) underwent training on various turbulent flow datasets followed by the Bayesian optimization for the neural network's hyper-parameters (i.e., the number of hidden layers, the number of nodes per hidden layer, and the gradient descent algorithm's learning rate). Therefore, Deep Learning architectures trained for mapping complex fluid flows can be optimized for detecting strange attractors within cancer networks.

Recurrent neural networks are sub-types of artificial neural networks that can accurately map the phase-space portraits of chaotic systems (Cestnik and Abel, 2019). While time-delay coordinate embedding is effective for the attractor reconstruction of low-dimensional systems, Reservoir Computing (RC) is a machine-learning algorithm that trains recurrent neural networks to find the Lyapunov exponents of high-dimensional datasets. For example, Reservoir Computing has demonstrated applicability in the chaotic attractor reconstruction of complex fluid flows (Nakai and Saiki, 2018). The RC well predicted the short-term time-series forecasting of the Kuramoto-Sivashinsky (KS) equation to several multiples of Lyapunov time. The KS equation is a chaotic system whose pattern formation closely resembles that of fluid turbulence (Pathak et al., $2018^{a, b}$ ). Hence, RC computing is a powerful class of machine learning algorithm that can be utilized on time-series cancer datasets to test the presence of strange attractors in data-driven science.

Lastly, algorithmic information dynamics (AID) is an emerging branch of computational complexity theory most pertinent to network biology (Zenil et al., 2019). AID combines tools from algorithmic information theory with perturbation analysis to study dynamical systems in software space (Zenil et al., 2019). While currently employed machine learning algorithms in the network reconstruction and pseudo-time ordering methods of gene expression datasets are based on statistical learning approaches, they do not inform us about cause and effect. The causality in cell fate transitions during cancer stem cell division and differentiation (pattern formation), and their underlying GRN information dynamics are not captured by current approaches cell lineage/trajectory inference and network visualization. They only perform pattern recognition on statistical features of the datasets heavily dependent on dimensionality reduction techniques and, often fail to treat the cells and networks as dynamical systems. As such, AID is a vastly unexplored artificial intelligence (AI) platform available for inferring causality in gene expression dynamics, cancer stem cell differentiation mapping and potentially map strange attractors in gene expression networks. 
To conclude, insights into chemical (fluid) turbulence and intracellular chaos within cells by means of complex systems tools such as algorithmic complexity measures and recurrent neural networks may pave the visualization of strange attractors in cancer datasets. In time, machine intelligence may be capable of assessing the causal information dynamics in cancer datasets and help us make clinically useful decisions from a complex systems perspective, at the emerging frontier of computational oncology.

"Big whorls have little whorls which feed on their velocity, and little whorls have lesser whorls and so on to viscosity"- L.F. Richardson (1922)

Figure 1: The Waddington landscape: The Waddington landscape shows a stem cell $\mathrm{S}$ bifurcating to various cell fates represented by the blue balls which are stuck in the basins of fixed-point attractors. As seen in red flow patterns, the gene expression underlying the differentiated cell states S1 and S2 seem more laminar (regular). However, cell fates are reversible as indicated by the dotted path integrals. Multiple bifurcation routes exist towards a local energy minimum $X$ which shows highly chaotic flows (in its underlying gene regulatory network or protein flow patterns). The attractor $\mathrm{X}$ is a chaotic cell fate (i.e., cancer stem cell). A cancer stem cell is shown as a strange attractor (in red) on the developmental landscape, with cell fate reprogramming towards the initial benign cell fate indicated by the two-way black arrow. Other possible strange-attractors are shown in various colored aperiodic, nonlinear orbits to demonstrate the proximity of multiple strange attractors for cancer-stem cell states and to show the difference of between a chaotic attractor and stable fixed-point attractors (blue balls).

ACKNOWLEDGEMENTS: Thanks to Dr. Mario D'Amico (Concordia University) for being my initial conditions.

DISCLOSURE STATEMENT: There are no competing interests. I, Abicumaran Uthamacumaran, am the sole author of the presented perspective. 


\section{REFERENCES}

1) Mitchell, M. Complexity: A guided tour. Oxford University Press (2009).

2) Thurner, S. et al., Introduction to the theory of complex systems. Oxford University Press. (2018)

3) Ladyman, J. and K. Wiesner, "What is a complex system?". Yale University Press (2020).

4) Strogatz, S.H. Nonlinear Dynamics and Chaos: With Applications to Physics, Biology, Chemistry, and Engineering (Studies in Nonlinearity). (2 ${ }^{\text {nd }}$ Ed.) (CRC Press, 2015)

5) Lorenz, E.N. Deterministic nonperiodic flow. J. Atm. Sci. 20:130-141 (1963)

6) Wensink, H. et al. Meso-scale turbulence in living fluids. PNAS, 109(36): 14308-13 (2012)

7) Arts \& Culture: Turbulence in The Starry Night. APS Physics. April 18, 2019• Physics 12, 45. https://physics.aps.org/articles/v12/45

8) Akutsu, T., Kuhara, S., Maruyama, O., and Miyano, S., A system for identifying genetic networks from gene expression patterns produced by gene disruptions and overexpressions. Genome Informatics 9, 151-160 (1998)

9) Milano, M. and Roli, A., Solving the satisfiability problem through Boolean networks. LNCS Vol. 1792. Advances in Al: 72-83. (2000)

10) Barillot, E. et al., Computational systems biology of cancer. CRC Press, Taylor \& Francis Group. (2013)

11) Songjian, L. et al. An exact algorithm for finding cancer driver somatic genome alterations: the weighted mutually exclusive maximum set cover problem. Algorithms for molecular biology: AMB vol. 11:11. (2016).

12) Hajkarim, M.C., Upfal, E. and Vandin, F. Differentially mutated subnetworks discovery. Algorithms for Molecular Biology volume 14, Article number: 10 (2019)

13) Ruelle, D. and Takens, F. On the nature of turbulence. Commun. Multi Phys. 20, 167-192 (1971)

14) Yankeelov, Thomas E et al. "Toward a science of tumor forecasting for clinical oncology." Cancer research vol. 75,6 (2015): 918-23.

15) Rössler, O.E., Chaotic behavior in simple reaction system, Zeitschrift für Naturforsch $A$, 31, 259-264, 1976 
16) Computation of bifurcation diagrams for Selkov's model of glycolytic oscillations. In L. Rensing and N. I. Jaeger (eds.): Temporal Order, pp. 197-200. Berlin: Springer, 1985

17) Heltberg, M.L. et al., On chaotic dynamics in transcription factors and the associated effects in differential gene regulation. Nature Communications 10, Article number: 71 (2019)

18) Itik, M. \& Banks, S.P., Chaos in a three-dimensional cancer model. international Journal of bifurcation and chaos. 20(01):71 -79 (2010)

19) Posadas, E.M. et al. Chaotic oscillations in cultured cells: rat prostate cancer. Cancer Res. 1996

20) Ivancevic, T.T. et al. A theoretical model of chaotic attractor in tumor growth and metastasis. arXiv:0807.4272 (2008) [q-Bio]

21) Khajanchi, S. et al. The influence of time delay in a chaotic cancer model. Chaos 28 , 103101 (2018)

22) Zheng $Q$, Shen J, Wang $Z$ (2018) Pattern dynamics of the reaction-diffusion immune system. PLoS ONE 13(1): e0190176.

23) Letellier, C. et al. What can be learned from a chaotic cancer model? J. Theor. Biol. 322: 7-16 (2013)

24) Shih, Hong-Yan et al. Ecological collapse and the emergence of travelling waves at the onset of shear turbulence. Nat. Phys. Vol 12: 245-248 (2016)

25) Ruelle, D. Strange attractors. Math. Intelligencer, vol 2: 126-137 (1980)

26) Ruelle, D. Turbulence, strange attractors and chaos. World Scientific Ser A. 16. (1995)

27) Brandstater, A. and Swinney, H.L., Strange attractors in weakly turbulent Couette-Taylor flow. Phys. Rev A. 35(5):2207-2220 (1987)

28) Lanford III, O.E., The strange attractor theory of turbulence. Ann rev FI Mech. 14:347-364 (1982)

29) Miles, J., Strange attractors in fluid dynamics. Adv App. Math. 24: 189-214 (1984)

30) Leray, J., Sur le mouvement dun liquide visqueux emplissant l'espace, Acta Math. 63 (1934)

31) Kolmogorov, A. N. Dissipation of energy in locally isotropic turbulence [Russian]. Dokl. Akad. Nauk SSSR 32, 16-18 (1941); English translation available in Levin, V. Proceedings: Mathematical and Physical Sciences (Turbulence and Stochastic Process: Kolmogorov's Ideas 50 Years On) Vol. 434 15-17 (Royal Society, 1991) 
32) Arnold, V. and Khesin, B.A. Topological methods in hydrodynamics. Springer 1991

33) Rico, R.L. (2000) An introduction to the geometry and topology of fluid flows. Kluwer Academic Publishers.

34) Ruelle, D. Some comments on chemical oscillations. Trans NewYork Acad. Sciences, Ser II. 35(1):66-71 (1973)

35) Brauns, F., Halatek, J. and Frey, E. Phase-space geometry of reaction-diffusion dynamics. arXiv:1812.08684 [nlin.PS] (2018)

36) Prigogine, I., Stengers, I. Order out of Chaos: Man's new dialogue with nature. Flamingo (1984)

37) Turing, A.M. The chemical basis of morphogenesis. Philos. Trans. R. Soc. Lond. B: Biol. Sci. 237 (641): 37-72 (1952)

38) Gatenby, RA and Gawlinki. A reaction-diffusion model of cancer invasion. Cancer Research 56(24):5745-53 (1996)

39) Ramis-Conde, I. et al. Mathematical modelling of cancer cell invasion of tissue. Math. Comp. Modelling. 47(5-6):533-545 (2008)

40) Domsuhke, P. et al. JTB. 361:41-60 (2014)- Mathematical modelling of cancer invasion: implications of cell adhesion variability for tumor infiltrative growth patterns.

41) Yan, H. et al. 3D Mathematical Modeling of Glioblastoma Suggests That Transdifferentiated Vascular Endothelial Cells Mediate Resistance to Current Standard-of-Care Therapy. Cancer Res; 77(15) (2017)

42) Hojin, H. et al. Age-Related Vascular Changes Affect Turbulence in Aortic Blood Flow. Frontiers in physiology vol. 9:36. (2018)

43) Ito, Y., et al. Turbulence activates platelet biogenesis to enable clinical scale ex vivo production. Cell. 174(3): 636-648 (2018)

44) Koumoutsakos, P. et al. The fluid mechanics of cancer and its therapy. Ann. Rev. Fl. Mech 45:325-355 (2013)

45) Goetz JG, Metastases go with the flow. Science 362(6418):999-1000 (2018)

46) Thamilselvan, V. and Basson, MD. Pressure activates colon cancer cell adhesion by inside- 
out focal adhesion complex and actin cytoskeletal signaling. Gastroenterology. 2004;126(1):8-18

47) Chivukula, V.K. et al. "Alterations in cancer cell mechanical properties after fluid shear stress exposure: a micropipette aspiration study". Cell health and cytoskeleton vol. 7 (2015): 25-35.

48) Rizvi, I. et al. "Flow induces epithelial-mesenchymal transition, cellular heterogeneity and biomarker modulation in 3D ovarian cancer nodules." PNAS vol. 110,22 (2013): E1974-83.

49) Ketene, AN et al. The effects of cancer progression on the viscoelasticity of ovarian cell cytoskeleton structures. Nanomedicine. Jan;8(1):93-102 (2012)

50) Huang, $Q$ et al. Fluid shear stress and tumor metastasis. American journal of cancer research vol. 8,5 763-777. (2018)

51) Baish, J.W. and Jain, R.K., Fractals and cancer. Canc. Res 60:3683-3688 (2000)

52) R. Nasu et al. Blood flow influences vascular growth during tumor angiogenesis. British Journal of Cancer (1999) 79(5/6), 780-786

53) Sreenivasan, K.R. and Meneveau, C. The fractal facets of turbulence. J Fluid Mech. Vol. 173: 357-386 (1986)

54) Sreenivasan, K.R. and Meneveau, C. The multifractal nature of turbulent energy dissipation. J. Fluid Mech. Vol 224: 429-484 (1991)

55) Coffey, WT. Self-organization, complexity and chaos: The new biology for medicine. Nat. Med. 4(8): 882-885 (1998)

56) Victor Yakhota and K.R. Sreenivasan, Towards a dynamical theory of multifractals in turbulence. Physica A 343 (2004) 147-155

57) Rejniak, K. A. "Circulating Tumor Cells: When a Solid Tumor Meets a Fluid Microenvironment." Advances in experimental medicine and biology vol. 936 (2016): 93-106.

58) Behr, J. et al. "Localized Modeling of Biochemical and Flow Interactions during Cancer Cell Adhesion." PloS one vol. 10,9 e0136926. (2015)

59) Yasuhara, K. et al. 25(4):183-8. New color Doppler technique for detecting turbulent tumor blood flow: a possible aid to hepatocellular carcinoma diagnosis. J Clin Ultrasound. (1997) 
60) Kalgin, I.V. \& Chekmarev, S.F. Turbulent phenomena in protein folding. Phys Rev E. $83,011920(2011)$

61) Andryushchenko, V.A. \& Chekmarev, S.F. Turbulence in protein folding: Vorticity, scaling and diffusion of probability flows. PLoS One. 12(12): e0188659 (2017)

62) Andryushchenko, V.A. and Chekmarev, S.F., On hydrodynamic interpretation of folding of an $\alpha$-helical protein. Thermophysics and Aeromechanics. Volume 23, Issue 6, pp 941-944 (2016)

63) Chekmarev, S.F. Protein Folding Dynamics in the Space of Experimentally Measured Variables: Turbulence Phenomena. J App Mech. Techn. Phys. Vol. 59, Issue 5, pp 827$833(2018)$

64) Sumino, Y. et al. "Large-scale vortex lattice emerging from collectively moving microtubules". Nature. 483 (7390): 448-452 (2012)

65) Doostmohammadi et al. Active nematics, Nature Communications. Volume 9, Article number: 3246 (2018)

66) Martinez-Pratt et al. Selection mechanism at the onset of active turbulence. Nature Physics, 15:362-366. (2019)

67) Marchetti, M.C. et al. Hydrodynamics of soft active matter. Rev Mod Phys. 85(3) (2013)

68) Baggaley, A.W., Stability of model flocks in a vortical flow. Phys. Rev. E 93, 063109 (2016)

69) Halatek, J. \& Frey, E. Rethinking pattern formation in reaction-diffusion systems. Nat. Phys. 14: 507-514 (2018) doi:10.1038/s41567-017-0040-5

70) Denk, J. et al., MinE conformational switching confers robustness on self-organized Min protein patterns. PNAS, 115 (18) 4553-4558. (2018).

71) Glock, P. et al., Design of biochemical pattern forming systems from minimal motifs. eLife. 8: e48646 (2019)

72) Kim, M. et al. Controlling Chemical Turbulence by Global Delayed Feedback: Pattern Formation in Catalytic CO Oxidation on Pt (110). Science. Vol. 292, Issue 5520, pp. 13571360 (2001)

73) K. R. Mecke. Morphological characterization of patterns in reaction-diffusion systems. Phys. Rev. E 53, 4794 (1996) 
74) Q Ouyang and H.L. Swinney, transition to chemical turbulence. Chaos, 1991. 1, 411

75) Ruelle, D. Hydrodynamic turbulence as a problem in nonequilibrium statistical mechanics. PNAS 109 (50) 20344-20346 (2012)

76) Ruelle, D. Nonequilibrium statistical mechanics of turbulence. J Stat Phys. 157(2), 205-208 (2014)

77) Takens, F., Detecting strange attractors in turbulence. Dynamical Systems and Turbulence, Warwick (1980)

78) Toker, D et al., A simple method for detecting chaos in nature. Comm. Biol. Vol. 3: 11 (2020)

79) Kutz, J.N. Deep learning in fluid dynamics. J. Fluid Mech. 814:1-4 (2017)

80) Yang, S. and Huang, Y. Complex dynamics in simple Hopfield neural networks. Chaos 16, 033114 (2016)

81) Mestl, T. et al., Chaos in high-dimensional neural and gene networks. Physica D. 98: 33-52 (1996)

82) Ling, J. and Templeton, J. Evaluation of machine learning algorithms for prediction of regions of high Reynolds averaged Navier Stokes uncertainty. Physics of Fluids, (2015)

83) Ling, J. et al. Reynolds averaged turbulence modelling using deep neural networks with embedded invariance. In: Journal of Fluid Mechanics 807 (2016), pp. 155-166.

84) Cestnik, R and Abel, M. Inferring dynamics of oscillatory systems using recurrent neural networks. Chaos 29, 063128 (2019)

85) Nakai, K. and Saiki, Y..Machine-learning inference of fluid variables from data using reservoir computing. Phys. Rev. E 98, 023111 (2018)

86) Pathak, J. et al. Hybrid forecasting of chaotic processes: using machine learning in conjunction with a knowledge-based model. Chaos 28(4) (2018)

87) Pathak, J. et al. Model-free prediction of larger spatiotemporally chaotic systems from data: a reservoir computing approach. Phys Rev. Lett. 120(2) (2018)

88) Zenil, H. et al., An algorithmic information calculus for causal discovery and reprogramming systems. iScience. 19, 1160-1172 (2019)

89) Zenil, H. et al., Causal deconvolution by algorithmic generative methods. Nature Machine Intelligence volume 1, pages 58-66(2019). 


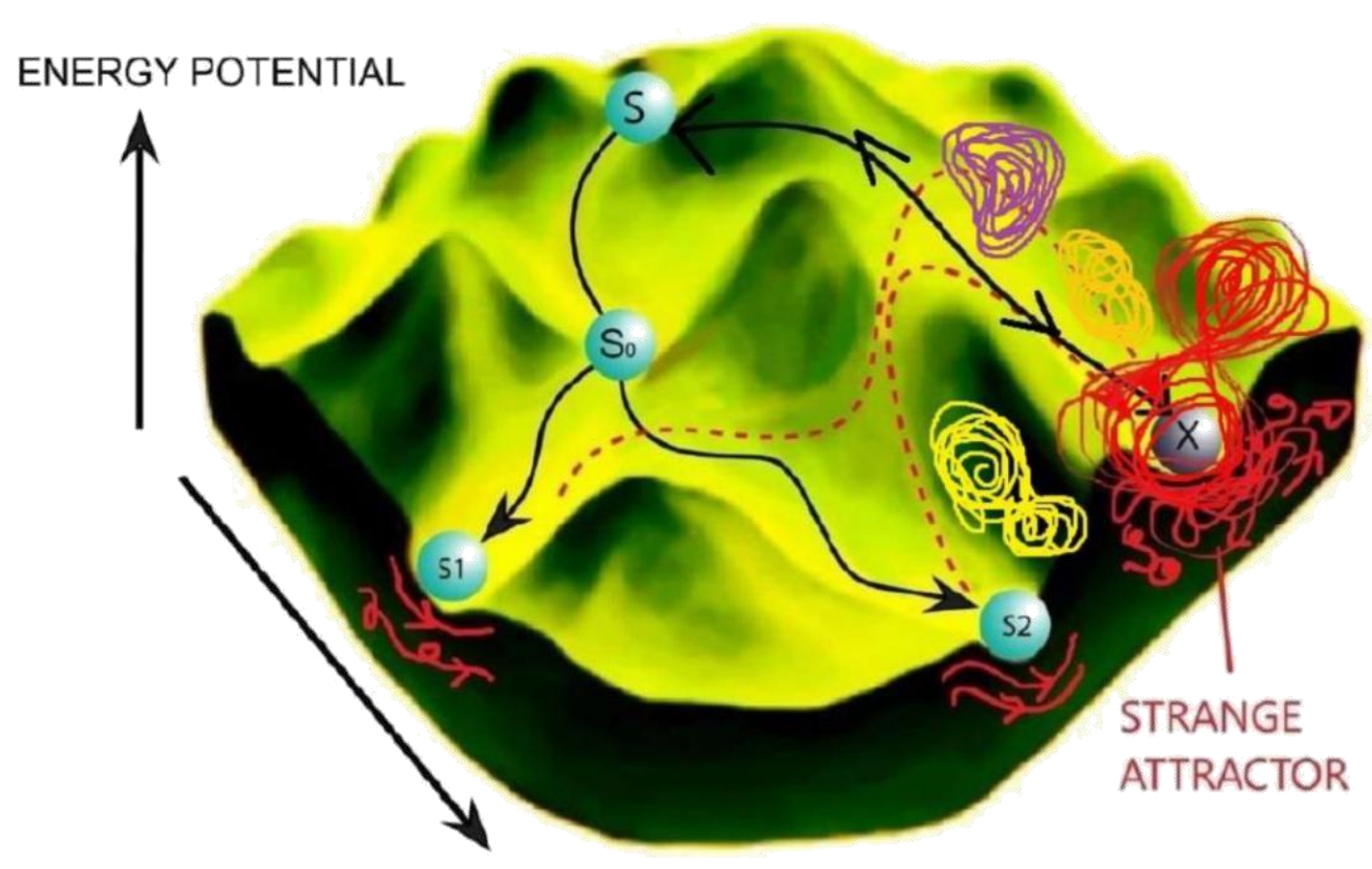

SPACE-TIME

FIGURE 1: THE WADDINGTON EPIGENETIC LANDSCAPE 